\title{
EFEITO DO ÓLEO DE SOJA NO CONTROLE DA ANTRACNOSE E NA CONSERVAÇÃO DA MANGA Cv. PALMER EM PÓS-COLHEITA ${ }^{1}$
}

\author{
NILTON TADEU VILELA JUNQUEIRA ${ }^{2}$, RENATA DA COSTA CHAVES ${ }^{3}$, ALESSANDRA CARNEIRO DO \\ NASCIMENTO3, VITOR HUGO VARGAS RAMOS², JOSÉ RICARDO PEIXOTO ${ }^{3}$, LÍVIA PEREIRA JUNQUEIRA ${ }^{3}$
}

\begin{abstract}
RESUMO - Na fase de pós-colheita da manga, a antracnose (Colletotrichum gloeosporioides Penz.) é a doença mais importante em termos de expressão econômica. Seu controle vem sendo feito pela imersão dos frutos por 5 minutos, em água a $55^{\circ} \mathrm{C}$, acrescida de thiabendazo1 a $0,2 \%$. Embora seja eficaz no controle dessa doença, esse fungicida pode deixar resíduo, o que não satisfaz os consumidores que vêm, a cada ano, aumentando as suas exigências por frutos livres de resíduos de agroquímicos e ambientalmente corretos. Dessa forma, esses experimentos foram conduzidos visando à seleção de produtos biológicos que tenham potencial para o controle da antracnose e para a conservação da manga na pós-colheita. Os frutos, colhidos no estádio de maturação $3 \mathrm{e} 4$, foram imersos por 5 minutos em thiabendazol a $0,24 \%$ e benomil a $0,1 \%$ a $22^{\circ} \mathrm{C}, 40^{\circ} \mathrm{C}$ ou $45^{\circ} \mathrm{C}$ e em diferentes concentrações de óleo de soja isolado ou em mistura com benomil, thiabendazol e com extrato etanólico de sucupira (Pterodon pubescens Benth.). Após os tratamentos, os frutos foram mantidos em câmaras a $27 \pm 1^{\circ} \mathrm{C}, 72 \%$ a $85 \%$ de UR (Experimento $n^{\circ} 1$ ) e a $17^{\circ} \mathrm{C}$ a $85 \%$ a $100 \%$ de UR (experimento $\mathrm{n}^{\mathrm{o}}$ 2). As avaliações foram efetuadas aos 15 dias (experimento $\mathrm{n}^{\mathrm{o}} 1$ ) e aos 30 dias (experimento $\mathrm{n}^{\mathrm{o}}$ 2) após os tratamentos, determinando-se as porcentagens da superfície dos frutos cobertas com lesões, de frutos verdes, maduros e de vez, ${ }^{\circ}$ Brix e textura. O óleo de soja, isolado ou misturado com benomil ou thiabendazol, a $22^{\circ} \mathrm{C}$ ou a $40^{\circ} \mathrm{C}$, aumentou o tempo de prateleira da manga Palmer e foi eficaz no controle da antracnose.
\end{abstract}

Termos para indexação: Mangifera indica, Colletotrichum gloeosporioides, controle alternativo, fitoterapia, extrato de Pterodon pubescens, defensivo biológico.

\section{EFFECT OF SOYBEAN OIL IN THE CONTROL OF ANTHRACNOSE AND ON POST-HARVEST CONSERVATION OF MANGO, Cv. PALMER}

\begin{abstract}
The anthracnose (Colletotrichum gloeosporioides Penz.) is the most important post-harvest disease of mango fruit. Its control has been done by immersion of fruits for 5 minutes in hot water at $55^{\circ} \mathrm{C}$ containing the fungicide thiabendazole at $200 \mathrm{~g} / 100$ liters. Although efficient in the control of mango anthracnose, this fungicide can leave residues. This fact does not satisfy the consumers who claim for pesticides-free fruits and pollution-free environment. Two experiments were carried out aiming at the selection of phytotherapeutics to control of post harvest anthracnose and to increase the mango shelf life period. Mango fruits were harvested in the $3 \mathrm{rd}$ and 4 th ripening stages, immersed for 5 minutes in water (control), benomyl $\left(100 \mathrm{~g} / 100\right.$ liters), thiabendazole $(240 \mathrm{~g} / 100$ liters $)$ at $22^{\circ} \mathrm{C}, 40^{\circ} \mathrm{C}$ or $45^{\circ} \mathrm{C}$ and in different concentrations of soybean oil alone or in mixtures with benomyl, thiabendazole and ethanolic extract of sucupira fruit (Pterodon pubescens Benth.). After treatments the fruits were maintained in room conditions at $27 \pm 1^{\circ} \mathrm{C}, \mathrm{RH}=72 \%-85 \%$ (Exp. n. 1) and at $17^{\circ} \mathrm{C}, \mathrm{RH}=85 \%-100 \%$ (Exp. n. 2). The experiments were evaluated at 15 days $\left(\right.$ Exp. $\left.\mathrm{n}^{\circ} 1\right)$ and 30 days after the treatment (Exp. $n^{\circ} 2$ ) by determining the percentages of the fruit area with lesions, ripe, half-ripe and unripe fruits, ${ }^{\circ} B r i x$ and texture. Soybean oil alone or with benomyl or thiabendazol at $22^{\circ} \mathrm{C}$ or $40^{\circ} \mathrm{C}$ increased mango shelf life period and was efficient in the control of anthracnose. Index Terms: Mangifera indica, Colletotrichum gloeosporioides, alternative control, phytotherapy, Pterodon pubescens extract, biologic pesticide
\end{abstract}

\section{INTRODUÇÃO}

Várias doenças acometem a manga na pós-colheita, provocando perdas expressivas. Entre essas, a antracnose causada por Colletotrichum gloeosporioides Penz é a mais expressiva (Junqueira et al., 2002a). No Brasil, o controle da antracnose e de outras doenças da manga em pós-colheita vem sendo feito pela imersão dos frutos durante 5 minutos, em água quente a $55^{\circ} \mathrm{C}$, acrescida de thiabendazol a $0,2 \%$ (Junqueira et al., 2002a). O benomil a $0,1 \%$ ou $0,2 \%$ também era utilizado e oferecia resultados satisfatórios, mas esse fungicida foi retirado do mercado por tempo indeterminado. Embora eficazes no controle dessa doença, esses fungicidas podem deixar resíduos nos frutos, o que não satisfaz os consumidores que, a cada dia, vêm aumentando suas exigências por frutos ambientalmente corretos e sem resíduos de agroquímicos (Junqueira et al., 2002a). Segundo Nascimento (2000) e Junqueira et al. (2000, 2002a, 2002b), a antracnose da manga, Cvs. Haden, Tommy Atkins e Winter, na pós-colheita, pode ser controlada com eficácia igual ou superior à de benomil e thiabendazol, pela imersão de seus frutos em caldas contendo extratos etanólicos de sucupira branca (Pterodon pubescens Benth. Sin. de P. emarginatus) a $20^{\circ} \mathrm{C}$ e $40^{\circ} \mathrm{C}$ e por água quente a $45^{\circ} \mathrm{C}$. Dessa forma, objetivando-se identificar produtos de origem biológica que tenham ação fungicida e que possam ser utilizados para reduzir a quantidade ou eliminar resíduos de fungicidas químicos em manga, conduziu-se o presente experimento, onde se avaliou a eficácia do óleo de soja e de extrato de sucupira no controle da antracnose e na conservação da manga na pós-colheita.

\section{MATERIALEMÉTODOS}

Foram conduzidos dois experimentos nas dependências da Embrapa Cerrados. Utilizaram-se mangas da Cv. Palmer produzidas num pomar localizado no Município de Sobradinho, Distrito Federal, colhidas nos estádios 3 e 4 (CIAD, A.C., 1998), nos meses de janeiro e fevereiro, um dia antes da instalação dos experimentos. As mangas não receberam tratamentos contra pragas e doenças na pré-colheita nem foram lavadas antes dos tratamentos.

No experimento número 1, utilizou-se delineamento experimental inteiramente casualizado, com treze tratamentos e 4 repetições de 9 frutos. Os frutos foram imersos durante cinco minutos, em caldas dos seguintes produtos: T1: Água a $22^{\circ} \mathrm{C}$; T2: Água a $45^{\circ} \mathrm{C}$; T3: benomil $1 \mathrm{~g} / 1.000 \mathrm{~mL}$ a $22^{\circ} \mathrm{C}$; T4: benomil $1 \mathrm{~g} / 1.000 \mathrm{~mL}$ a $45^{\circ} \mathrm{C}$; $\mathrm{T} 5$ : thiabendazol (TBZ) $2,4 \mathrm{~g} /$ $1.000 \mathrm{~mL}$ a $22^{\circ} \mathrm{C}$; $\mathrm{T} 6$ : $\mathrm{TBZ} 2,4 \mathrm{~g} / 1.000 \mathrm{~mL} \mathrm{a} 45^{\circ} \mathrm{C}$; $\mathrm{T} 7: 30 \mathrm{~mL}$ de extrato concentrado de sucupira $+25 \mathrm{ml}$ de óleo de soja $/ 1.945 \mathrm{~mL}$ de água a $22^{\circ} \mathrm{C}$ ; T8: $30 \mathrm{~mL}$ de extrato concentrado de sucupira $+25 \mathrm{~mL}$ de óleo de soja/ $1.945 \mathrm{~mL}$ de água a $40^{\circ} \mathrm{C} ; \mathrm{T} 9: 100 \mathrm{~mL}$ de óleo de soja $+36 \mathrm{~g}$ de leite em pó instantâneo (LPI)/ 1.900mL de água; T10: $50 \mathrm{~mL}$ de óleo de soja $+36 \mathrm{~g}$ de LPI/ 1.950mL de água ; T11: $50 \mathrm{~mL}$ de óleo de soja $+36 \mathrm{~g}$ de LPI $+2 \mathrm{~g}$ de benomil/1.950mL de água a $40^{\circ} \mathrm{C}$; T12: $50 \mathrm{~mL}$ de óleo de soja $+36 \mathrm{~g}$ de LPI /1.950mL de água a $40^{\circ} \mathrm{C}$ e T13: $50 \mathrm{~mL}$ de óleo de soja $+36 \mathrm{~g}$ de LPI / $1.950 \mathrm{~mL}$ de água a $40^{\circ} \mathrm{C}+4,8 \mathrm{~g}$ de $\mathrm{TBZ}$.

Imediatamente após os tratamentos, os frutos foram acondicionados em caixas de plástico e armazenados a $27 \pm 1^{\circ} \mathrm{C}$ e umidade relativa de 72 a $85 \%$. As avaliações foram efetuadas aos 15 dias após os

\footnotetext{
${ }^{1}$ (Trabalho 181/2003). Recebido: 04/12/2003. Aceito para publicação: 14/06/2004.

${ }^{2}$ Embrapa Cerrados, Cx. Postal 08223, CEP 73301-970, Planaltina-DF, Tel. 0xx 61-3889829 E-mail: junqueir@cpac.embrapa.br ; vhugo@cpac.embrapa.br

${ }_{3}^{3}$ Faculdade de Agronomia e Veterinária, Universidade de Brasília, CEP 70910-970, Brasília-DF, Tel. 0xx 61 307- 2801. E-mail: peixoto@unb.br, renatachaves@unb.br
} 
TABELA 1 - Porcentagem de frutos lesados e da área dos frutos da Cv. Palmer coberta com lesões causadas por Colletotrichum gloeosporioides, Brix, textura, porcentagem de frutos murchos, porcentagem de frutos maduros, de vez e verdes, aos 15 dias após os tratamentos, Brasília, 2002.

\begin{tabular}{|c|c|c|c|c|c|c|c|}
\hline Tratamentos & $\begin{array}{c}\text { Frutos lesados } \\
(\%)\end{array}$ & $\begin{array}{c}\text { Área do Fruto } \\
\text { coberta com Lesões } \\
(\%)\end{array}$ & ${ }^{\mathbf{o}} \mathrm{Brix}$ & $\begin{array}{l}\text { Textura } \\
\left(\mathrm{gf} / \mathrm{cm}^{2}\right)\end{array}$ & $\begin{array}{c}\text { Frutos maduros } \\
(\%)\end{array}$ & $\begin{array}{l}\text { Frutos de } \\
\text { Vez }(\%)\end{array}$ & $\begin{array}{c}\text { Frutos } \\
\text { Verdes } \\
(\%) \\
\end{array}$ \\
\hline $\mathrm{T} 1$ & $100 \mathrm{a}$ & $73,39 \mathrm{a}$ & $14,00 \mathrm{a}$ & $318,94 b$ & $54 a$ & $46 a$ & $0 \mathrm{~b}$ \\
\hline $\mathrm{T} 2$ & $100 \mathrm{a}$ & $75,73 \mathrm{a}$ & $11,75 \mathrm{a}$ & $416,56 a$ & $57 \mathrm{a}$ & $43 a$ & $0 \mathrm{~b}$ \\
\hline T3 & $33 c$ & $12,03 \mathrm{def}$ & $10,25 \mathrm{a}$ & $449,44 a$ & $48 \mathrm{a}$ & $52 \mathrm{a}$ & $0 \mathrm{~b}$ \\
\hline $\mathrm{T} 4$ & $2 d$ & $1,17 f$ & $14,38 \mathrm{a}$ & $440,94 a$ & $55 \mathrm{a}$ & $45 \mathrm{a}$ & $0 \mathrm{~b}$ \\
\hline $\mathrm{T} 5$ & $86 a$ & $62,33 a$ & $10,13 a$ & $445,50 \mathrm{a}$ & $57 \mathrm{a}$ & $43 a$ & $0 \mathrm{~b}$ \\
\hline T6 & $53 b$ & $25,39 \mathrm{bcd}$ & $14,00 \mathrm{a}$ & $438,00 \mathrm{a}$ & $38 \mathrm{ab}$ & $47 \mathrm{a}$ & $16 \mathrm{ab}$ \\
\hline $\mathrm{T} 7$ & $36 \mathrm{c}$ & $18,16 \mathrm{cde}$ & $10,44 a$ & $446,56 a$ & $3 b$ & $44 a$ & $53 a$ \\
\hline $\mathrm{T} 8$ & $64 \mathrm{ab}$ & $35,90 b$ & $12,19 \mathrm{a}$ & $453,88 \mathrm{a}$ & $34 \mathrm{ab}$ & $43 a$ & $20 \mathrm{ab}$ \\
\hline T9 & $41 \mathrm{c}$ & $17,60 \mathrm{cde}$ & $8,56 \mathrm{a}$ & $458,06 a$ & $12 \mathrm{ab}$ & $45 \mathrm{a}$ & $43 a$ \\
\hline $\mathrm{T} 10$ & $56 \mathrm{ab}$ & $36,96 b c$ & $10,38 \mathrm{a}$ & $464,06 a$ & $3 b$ & $56 a$ & $41 \mathrm{a}$ \\
\hline $\mathrm{T} 11$ & $2 d$ & $0,48 \mathrm{f}$ & $13,25 \mathrm{a}$ & $463,81 \mathrm{a}$ & $33 \mathrm{ab}$ & $64 \mathrm{a}$ & $3 b$ \\
\hline $\mathrm{T} 12$ & $36 c$ & $21,91 \mathrm{cde}$ & $10,00 \mathrm{a}$ & $459,00 \mathrm{a}$ & $22 \mathrm{ab}$ & $36 a$ & $42 a$ \\
\hline $\mathrm{T} 13$ & $3 d$ & $7,28 \mathrm{ef}$ & $11,81 \mathrm{a}$ & $449,63 a$ & $14 \mathrm{ab}$ & $50 \mathrm{a}$ & $36 a$ \\
\hline C.V. & 18,20 & 21,60 & 22,00 & 5,10 & 35,20 & 22,10 & 25,30 \\
\hline
\end{tabular}

Médias seguidas por diferentes letras diferem entre si, pelo teste deTukey, ao nível de $1 \%$.

Os dados em \% foram transformados para arco-senov\%

Tratamentos: $\mathbf{T 1}=$ Água a $22^{\circ} \mathrm{C} ; \mathbf{T} 2=$ Água a $45^{\circ} \mathrm{C} ; \mathbf{T 3}=$ benomil $1 \mathrm{~g} / 1$ a $22^{\circ} \mathrm{C} ; \mathbf{T} 4=$ benomil $1 \mathrm{~g} / 1$ a $45^{\circ} \mathrm{C} ; \mathbf{T 5}=\mathrm{TBZ} 2,4 \mathrm{~g} / 1$ a $22^{\circ} \mathrm{C} ; \mathbf{T 6}=\mathrm{TBZ} 2,4 \mathrm{~g} / 1$ a $45^{\circ} \mathrm{C} ; \mathbf{T} 7$ $=30 \mathrm{ml}$ de extrato de sucupira $+25 \mathrm{ml}$ de óleo de soja $/ 1.945 \mathrm{ml}$ de água a $22^{\circ} \mathrm{C} ; \mathbf{T 8}=\mathrm{T} 7 \mathrm{a} 40^{\circ} \mathrm{C} ; \mathbf{T} 9=100 \mathrm{ml}$ de óleo de soja $+36 \mathrm{~g}$ de leite em pó $/ 1.900 \mathrm{ml}$ de água; $\mathbf{T 1 0}=50 \mathrm{ml}$ de óleo de soja $+36 \mathrm{~g}$ de leite em pó $/ 1.950 \mathrm{ml}$ de água; $\mathbf{T 1 1}=50 \mathrm{ml}$ de óleo de soja $+36 \mathrm{~g}$ de leite em pó $+2 \mathrm{~g}$ de benomil $/ 1.950 \mathrm{ml}$ de água a $40^{\circ} \mathrm{C} ; \mathbf{T 1 2}$ $=50 \mathrm{ml}$ de óleo de soja $+36 \mathrm{~g}$ de leite em pó $/ 1.950 \mathrm{ml}$ de água a $40^{\circ} \mathrm{C}$, e $\mathbf{T 1 3}=\mathrm{T} 12+4,8 \mathrm{~g}$ de $\mathrm{TBZ}$.

Os frutos foram mantidos por 15 dias à temperatura de $27 \pm 1^{\circ} \mathrm{C}$ e umidade relativa de $72 \%$ a $85 \%$

tratamentos, determinando-se a percentagem da superfície dos frutos com antracnose, de frutos lesados, de frutos maduros, frutos de vez, frutos verdes, Brix e textura dos frutos determinada por meio de um texturômetro Teclock. Para determinar os percentuais da superfície dos frutos cobertos com antracnose, determinaram-se a área lesada com base no número de lesões por fruto e o diâmetro médio dessas lesões e a média das áreas de 20 frutos colhidos ao acaso. Para tal, as cascas foram retiradas de 20 frutos, cortadas em pequenos retângulos e dispostas sobre uma superfície plana, onde a área média foi determinada.

Para a obtenção do extrato concentrado de sucupira, foi utilizada a metodologia descrita por Junqueira et al. (2000) e Nascimento (2000), na qual 1.000 gramas de frutos de sucupireira branca, desaletados, foram moídos e colocados em $2.000 \mathrm{~mL}$ de etanol e mantidos a $23^{\circ} \mathrm{C}$ por 5 dias. Após a coagem, retiraram-se $1.000 \mathrm{~mL}$ de extrato que foram concentrados por evaporação do etanol, a $22^{\circ} \mathrm{C}$, até atingir o volume de $350 \mathrm{~mL}$

O leite em pó instantâneo (LPI) foi utilizado como emulsificante natural para o óleo de soja, pelo fato de este conter, em sua formulação, a lecitina de soja e estar disponível em qualquer mercado. O extrato de sucupira, por conter resinas, precisa ser misturado ao óleo vegetal para que seu manuseio seja facilitado.

Embora na literatura conste a utilização de água a $55^{\circ} \mathrm{C}$ nos tratamentos de manga em pós-colheita, nestes experimentos, utilizou-se água a $45^{\circ} \mathrm{C}$ pelo fato de temperaturas mais elevadas terem provocado injúrias nos frutos produzidos no Distrito Federal.

No Experimento número 2, aplicaram-se os seguintes tratamentos: $\mathrm{T} 1$ : Água a $22^{\circ} \mathrm{C}$; $\mathrm{T} 2$ : benomil $1 \mathrm{~g} / 1.000 \mathrm{~mL}$ a $45^{\circ} \mathrm{C}$; $\mathrm{T} 3$ : $50 \mathrm{~mL}$ de óleo de soja $+36 \mathrm{~g}$ de leite em pó instantâneo (LPI) $+2 \mathrm{~g}$ de benomil $/ 1.950 \mathrm{~mL}$ de água a $40^{\circ} \mathrm{C} ; \mathrm{T} 4: 50 \mathrm{~mL}$ de óleo de soja $+36 \mathrm{~g} \mathrm{de}$ $\mathrm{LPI}+4,8 \mathrm{~g}$ de TBZ/ $1.950 \mathrm{~mL}$ de água a $40^{\circ} \mathrm{C}$; T5: $100 \mathrm{~mL}$ de óleo de soja $+36 \mathrm{~g}$ de LPI/ $1.900 \mathrm{~mL}$ de água. As frutas foram armazenadas em caixas de plástico sob temperatura controlada a $17^{\circ} \mathrm{C}$ e umidade relativa de $85-100 \%$. O delineamento experimental foi inteiramente casualizado, com 5 tratamentos e 4 repetições de 9 frutos. As avaliações foram efetuadas aos 30 dias após os tratamentos, determinando-se os percentuais da superfície dos frutos cobertas com antracnose, e de frutos lesados, e os percentuais de frutos maduros, frutos de vez e frutos verdes.

\section{RESULTADOS E DISCUSSÃO}

A antracnose foi a principal doença que apareceu nos frutos. Em alguns casos, constatou-se a presença da podridão-parda causada por Dothiorella dominicana.

Para o primeiro experimento (Tabela 1), verifica-se que, aos 15 dias após, os tratamentos mais eficazes no controle da antracnose foram: T11 (50 mL de óleo de soja $+36 \mathrm{~g} \mathrm{LPI}+2 \mathrm{~g}$ de benomil $/ 1.950 \mathrm{~mL}$ de água com pH 5,6, aquecida a $40^{\circ} \mathrm{C}$ ); $\mathrm{T} 4$ (benomil $2 \mathrm{~g} / 2.000 \mathrm{mLa} 45^{\circ} \mathrm{C}$ ), e T13 (50 $\mathrm{mL}$ de óleo de soja $+36 \mathrm{~g}$ de LPI $+4,8 \mathrm{~g}$ de TBZ/ $1.950 \mathrm{~mL}$ de água aquecida a $40^{\circ} \mathrm{C}$ ). Os tratamentos T3, T9, T7, T12, T6, T8 e T10 à base de óleo de soja + leite em pó, extrato de sucupira aquecido ou não, TBZ aquecido e benomil foram muito eficazes quando comparados às testemunhas (água a $22^{\circ} \mathrm{Ce} 45^{\circ} \mathrm{C}$ ) e $\mathrm{TBZ}$ a $22^{\circ} \mathrm{C}$.

Não houve diferenças estatísticas para ${ }^{\circ}$ Brix entre os tratamentos. Os frutos do tratamento $\mathrm{T} 1$ apresentaram a textura menos firme, não havendo diferenças significativas entre os demais tratamentos. Não houve diferenças estatísticas no percentual de frutos de vez, mas, quanto aos frutos maduros e verdes, houve diferenças altamente significativas. Os tratamentos $\mathrm{T} 7$ ( $30 \mathrm{~mL}$ de extrato de sucupira $+25 \mathrm{~mL}$ de óleo de soja/1.945mL) e T10 ( $50 \mathrm{~mL}$ de óleo de soja $+36 \mathrm{~g}$ de leite em pó $/ 1.950 \mathrm{~mL}$ ) apresentaram o menor percentual $(3 \%)$ de frutos maduros, ao passo que os tratamentos de T1 a T5 induziram o maior percentual de frutos maduros (48\% a 57\%). Quanto aos frutos verdes, os tratamentos T7, T9, T12 e T10 mantiveram o maior percentual (41\% a 53\%). Não foram observados frutos verdes nos tratamentos de T1 a T5.

Esses resultados evidenciam o potencial do óleo de soja e do extrato de sucupira no controle da antracnose e na conservação de mangas na pós-colheita, conforme já constatado por Junqueira et al. (2000) e Nascimento (2000). Estes autores, trabalhando com frutos das cultivares Haden, Winter e Kent, verificaram que o extrato de sucupira puro (não acrescido de óleo soja) a $22^{\circ} \mathrm{C}$ e a $40^{\circ} \mathrm{C}$, benomil a $1 \mathrm{~g} /$ litro a 45 ${ }^{\circ} \mathrm{C}$ e água quente a $45^{\circ} \mathrm{C}$ foram eficazes no controle da antracnose em pós-colheita, em avaliações efetuadas aos 15 dias após a colheita. Estes autores verificaram que benomil e thiabendazol a $22^{\circ} \mathrm{C}$ não controlaram a doença. Por outro lado, analisando a Tabela 1 , verifica-se que, com os frutos da cultivar Palmer, o benomil a $1 \mathrm{~g} /$ litro a $22^{\circ} \mathrm{C}$ foi eficaz no controle 
da antracnose, ao passo que a água quente a $45^{\circ} \mathrm{C}$ não foi eficaz. Essa diferença pode estar relacionada com a espessura da casca, cerosidade e com a diferença de suscetibilidade entre essas cultivares, ao Colletotrichum gloeosporioides. Nascimento (2000) verificou, também, que o extrato concentrado de sucupira, diluído em 20 vezes, inibiu de forma eficaz, o crescimento in vitro de Colletotrichum gloeosporioides em meio de cultura à base de batata, dextrose e Ágar. Vários autores (Innecco, 2003; Mota et al., 2000; Moura et al., 2000) relataram a eficácia, também, de óleos essenciais (óleos de origem vegetal, que contêm substâncias aromáticas, geralmente utilizados na fabricação de cosméticos) na inibição do crescimento in vitro de agentes patogênicos.

Quanto ao efeito de óleos vegetais in vivo, Silva et al. (2002) verificaram que óleos vegetais, extrato de sucupira e outros produtos biológicos aumentaram o tempo de conservação da banana na póscolheita e foram eficazes no controle da antracnose. Resultados semelhantes foram obtidos por Junqueira et al. (2003) trabalhando com pós-colheita de mamão-papaia e por Junqueira et al. (2002b) trabalhando com manga.

No segundo experimento (Tabela 2), verifica-se que, após 30 dias de armazenamento em câmara a $17^{\circ} \mathrm{C}$ e umidade relativa variando de $85 \%$ a $100 \%$, os frutos imersos na calda à base de $50 \mathrm{~mL}$ de óleo de soja $+36 \mathrm{~g}$ de leite em pó $+2 \mathrm{~g}$ de benomil/1.950 $\mathrm{mL}$ de água com $\mathrm{pH}=5,6$ apresentaram o menor índice de antracnose, ou seja, de 6,0\% contra $89,28 \%$ na testemunha, o menor percentual de frutos maduros $(2,78 \%) \mathrm{e}$ o maior de frutos verdes $(66,67 \%)$. O T2, à base de benomil a $1 \mathrm{~g} / 1.000 \mathrm{~mL}$, aquecido a $45^{\circ} \mathrm{C}$, propiciou o mesmo nível de controle da antracnose obtido com os tratamentos à base de $50 \mathrm{~mL}$ de óleo de soja $+36 \mathrm{~g}$ de leite em pó $+4,8 \mathrm{~g}$ de TBZ/1.950 $\mathrm{mL}$ de água a $40{ }^{\circ} \mathrm{C}(\mathrm{T} 4)$ e $100 \mathrm{~mL}$ de óleo de soja $+36 \mathrm{~g}$ de leite em pó/1.900 $\mathrm{mL}$ de água a $22^{\circ} \mathrm{C}(\mathrm{T} 5)$. Analisando a Tabela 2, verifica-se que todos os tratamentos contendo óleo de soja controlaram bem a antracnose e impediram o amadurecimento dos frutos em comparação com os tratamentos contendo somente o benomil, TBZ e testemunha. No entanto, verificou-se que o processo de amadurecimento dos frutos foi bloqueado pelo óleo de soja e não houve reversão, mesmo após os frutos terem sido armazenados a $27 \pm 1^{\circ} \mathrm{C}$ por uma semana. Esses frutos, embora sadios e com boa aparência, estavam com um ligeiro sabor e aroma de álcool, indicando a existência de algum processo de fermentação interna. Dessa forma, há necessidade de pesquisas nessa área visando a determinar estádios de maturação ideal e à obtenção de técnicas ou produtos que possam reverter o bloqueio do processo de amadurecimento de frutos tratados com óleos vegetais, de forma isolada ou misturado com fungicidas. Não há, na literatura, informações sobre a ação de óleos vegetais no controle de doenças e na conservação de frutos em pós-colheita, mas acredita-se que o óleo bloqueia, de forma parcial ou total, a respiração do fruto. Conseqüentemente, pode não haver produção de etileno e conversão do amido em açucares, impedindo ou retardando o desenvolvimento dos patógenos. Segundo Chitarra \& Chitarra (1990), após a colheita, o principal processo fisiológico do fruto é a respiração. Neste caso, ele adquire vida independente, utilizando, para isso, suas reservas acumuladas. O tipo e a intensidade da atividade fisiológica na pós-colheita determinam a sua longevidade. Conforme citado por Pereira \& Beltran (2002) e Finger \& Vieira (2002), o processo de amadurecimento do fruto é dependente do etileno. Para que sua síntese ocorra, é necessária a presença do oxigênio, que é um dos substratos da enzima ACC ( 1-ácido carboxílico 1 aminociclopropano oxidase). Dessa forma, acredita-se que o óleo vegetal forme um filme sobre a epiderme do fruto, reduzindo as trocas gasosas e, conseqüentemente, diminuindo a concentração do oxigênio intracelular.

Quanto à composição do extrato etanólico de sucupira, Machado e Peixoto (1938), citados por Nascimento (2000), relatam a presença de terpenos e sesquiterpenos, sendo $89,30 \%$ de cedreno, $10,10 \%$ de 1 -alfa cariofileno e $0,42 \%$ de cariofoleno, além de ácido fenilacrílico, ácidos resínicos, álcool resínico e resenos.

Quanto à toxicidade para humanos, o extrato de sucupira é tradicionalmente utilizado na medicina popular para tratamento de amigdalite, reumatismo (Almeida et al., 1998) e como cicatrizante. No entanto, acredita-se que sua toxicidade para mamíferos não seja elevada caso venha a ser utilizado como defensivo agrícola.

TABELA 2 - Porcentagem da área dos frutos coberta com lesões causadas por Colletotrichum gloeosporioides, de frutos lesados, de frutos maduros, frutos de vez e frutos verdes da cultivar Palmer, aos 30 dias após os tratamentos. Brasília, 2002.

\begin{tabular}{cccccc}
\hline Tratamento & $\begin{array}{c}\text { Frutos } \\
\text { lesados } \\
(\%)\end{array}$ & $\begin{array}{c}\text { Área do } \\
\text { fruto } \\
\text { coberta com } \\
\text { lesões (\%) }\end{array}$ & $\begin{array}{c}\text { Frutos } \\
\text { maduros } \\
\mathbf{( \% ) ~})\end{array}$ & $\begin{array}{c}\text { Frutos } \\
\text { de Vez } \\
\mathbf{( \% )}\end{array}$ & $\begin{array}{c}\text { Frutos } \\
\text { Verdes } \\
\mathbf{( \% )}\end{array}$ \\
\hline T 1 & $100,00 \mathrm{a}$ & $89,28 \mathrm{a}$ & $79,91 \mathrm{a}$ & $20,08 \mathrm{a}$ & $0,00 \mathrm{~b}$ \\
T 2 & $53,35 \mathrm{~b}$ & $33,64 \mathrm{~b}$ & $61,11 \mathrm{a}$ & $38,89 \mathrm{a}$ & $0,00 \mathrm{~b}$ \\
T 3 & $4,23 \mathrm{c}$ & $6,00 \mathrm{c}$ & $2,78 \mathrm{~b}$ & $30,56 \mathrm{a}$ & $66,67 \mathrm{a}$ \\
T 4 & $52,00 \mathrm{~b}$ & $36,89 \mathrm{~b}$ & $11,11 \mathrm{~b}$ & $41,67 \mathrm{a}$ & $47,22 \mathrm{a}$ \\
T 5 & $42,60 \mathrm{~b}$ & $28,46 \mathrm{~b}$ & $16,67 \mathrm{~b}$ & $55,56 \mathrm{a}$ & $27,78 \mathrm{a}$ \\
\hline C.V. & 19,62 & 20,97 & 23,00 & 26,10 & 24,62 \\
\hline
\end{tabular}

Médias seguidas por diferentes letras diferem entre si, pelo teste de Tukey, ao nível de $1 \%$. Os dados foram transformados para arco-senov\% Tratamentos: $\mathbf{T 1}=$ Água a $22^{\circ} \mathrm{C} ; \mathbf{T} 2=$ benomil $1 \mathrm{~g} / \mathrm{l} \mathrm{a} 45^{\circ} \mathrm{C} ; \mathbf{T} 3=50 \mathrm{ml}$ de óleo de soja $+36 \mathrm{~g}$ de leite em pó $+2 \mathrm{~g}$ de benomil $/ 1.950 \mathrm{ml}$ de água a $40^{\circ} \mathrm{C}$; T4 = 50ml de óleo de soja $+36 \mathrm{~g}$ de leite em pó $+4,8 \mathrm{~g}$ de TBZ/1.950ml de água a $40^{\circ} \mathrm{C} ; \mathbf{T 5}=100 \mathrm{ml}$ de óleo de soja $+36 \mathrm{~g}$ de leite em pó $/ 1.900 \mathrm{ml}$ de água a $22^{\circ} \mathrm{C}$.

Os frutos foram mantidos por 30 dias em câmara a $17^{\circ} \mathrm{C}$ e umidade relativa de $85 \%$ a $100 \%$

\section{CONCLUSÕES}

1. O óleo de soja, isolado ou misturado com benomil ou thiabendazol, a $22^{\circ} \mathrm{C}$ ou a $40^{\circ} \mathrm{C}$, aumentou o tempo de prateleira da manga Palmer e foi eficaz no controle da antracnose.

2. Os frutos tratados com óleo de soja e armazenados em câmara a $17^{\circ} \mathrm{Ce} 85$ a $100 \%$ de umidade relativa permaneceram verdes ou de vez por um período de 30 dias, mas não amadureceram quando a temperatura da câmara foi elevada para $27 \pm 1^{\circ} \mathrm{C}$.

3. O thiabendazol não controlou a doença, mostrando-se eficiente apenas quando adicionado ao óleo de soja ou quando aquecido. óleo de soja.

4. O benomil foi mais eficaz quando aquecido ou misturado ao

5. Os fungicidas, na ausência do óleo de soja, não tiveram efeito na conservação dos frutos.

6. O extrato de sucupira foi eficaz na conservação dos frutos e no controle da doença, mas, nas concentrações utilizadas, provocou leves queimaduras.

\section{REFERÊNCIAS BIBLIOGRÁFICAS}

ALMEIDA, S.P.; PROENÇA, C.E.B.; SANO, S.M.; RIBEIRO, J.F. Cerrado: espécies vegetais úteis. Planaltina: EMBRAPA-CPAC, 1998. 464p.

CIAD, A.C. Centro de Investigaçion en Alimentacion y Desarrollo, A.C. Norma de Calidad para Mango Fresco de Exportación. Zapopan, Jalisco, Enero de1998.

CHITARRA, M.I.F.; CHITARRA, A.B. Pós-colheita de frutos e hortaliças: fisiologia e Manuseio. Lavras, MG, ESAL-FAEP, 320p. 1990.

FINGER, F.L.; VIEIRA, G. Fisiologia pós-colheita de frutos tropicais e subtropicais. In: ZAMBOLIM, L. (Org.) Manejo integrado: fruteiras tropicais-doenças e pragas. Viçosa: MG: UFV, 2002a. v.1, p. 1-30. il.

INNECCO, R. Efeito de óleos essenciais de plantas medicinais como defensivo agrícola. Fitopatologia Brasileira, Brasília, v.28, suplemento, p. S 57, 2003.

JUNQUEIRA, N.T.V.; SILVA. A. de O.; CHAVES, R.C. da. Efeito do óleo de soja no controle da antracnose (Colletotrichum gloeosporioides) e na maturação do mamão-papaia na pós-colheita. Fitopatologia 
Brasileira, Brasília, v.28, supl. p. S 352, 2003, (Resumo 698).

JUNQUEIRA, N.T.V.; PINTO, A.C.de Q.; CUNHA, M.M.; RAMOS, V.H.V.

Controle das doenças da mangueira. In: ZAMBOLIM, M.L. (Org.) Controle de Doenças de Plantas: fruteiras. Viçosa-MG: UFV, 2002a. v.1, p. 323-404.

JUNQUEIRA, N.T.V.; CHAVES, R.C.; PINTO, A.C.de Q.; RAMOS, V.H.V.; FIALHO, J.F.F. Efeito do óleo de soja e de outros produtos naturais no controle de doenças e na conservação da manga Palmer em póscolheita. In: CONGRESSO BRASILEIRO DE FRUTICULTURA, 17. 2002, Belém, Anais... CD-ROM.

JUNQUEIRA, N. T. V.; NASCIMENTO, A.C. do; PINTO, A.C. de Q.; RAMOS, V.H.V.; PIO, R.; RANGEL, L.E.P.; SILVA, J.A. da; FIALHO, J.F. Efeito do extrato dos frutos de sucupira-branca (Pterodon pubescens Benth.) e de outros produtos naturais no controle de doenças de manga na pós-colheita. In: CONGRESSO BRASILEIRO DE DEFENSIVOS AGRÍCOLAS NATURAIS, 1., 2000, Fortaleza, Anais...

MOTA, J.C.O.; PESSOA, M.N.G.; ANDRADE NETO, M. Efeito de óleos essenciais sobre o crescimento micelial de Lasiodiplodia theobromae, "in vitro". In: CONGRESSO BRASILEIRO DE
DEFENSIVOS AGRÍCOLAS NATURAIS, 1., 2000, Fortaleza. Anais... MOURA, J.S.; FEITOSA, V.S.; PESSOA, M.N.G. Efeito de óleos essenciais sobre o crescimento micelial de Colletotrichum gloeosporioides, Lasiodiplodia theobromae e Macrophomina phaseolina " in vitro". In: CONGRESSO BRASILEIRO DE DEFENSIVOS AGRÍCOLAS NATURAIS, 1., 2000, Fortaleza, Anais...

NASCIMENTO, A. C. do. Efeito de defensivos agrícolas naturais no controle de doenças da manga (Mangifera indica L.) na pós-colheita. 2000. 59f. il. Monografia (Trabalho de graduação em Agronomia), Faculdade de Agronomia e Veterinária, Universidade de Brasília, Brasília, 2000.

PEREIRA, W.S.P.; BELTRAN, A. Mecanismo de ação e uso do 1-MCP, bloqueador da ação do etileno visando prolongar a vida útil das frutas. In: ZAMBOLIM, M. L. (Org.), Manejo integrado: fruteiras tropicais-doenças e pragas. Viçosa - MG: UFV, 2002. v.1, p. 31-46.

SILVA, A. P.de O.; JUNQUEIRA, N.T.V.; CHAVES, R. da C.; FIALHO, J.F.; JUNQUEIRA, L.P. Efeito de defensivos naturais no controle da antracnose e na conservação de bananas na pós-colheita. In: CONGRESSO BRASILEIRODE FRUTICULTURA, 17,. 2002, Belém. Anais... CD-ROM. 